\title{
BMJ Open C-reactive protein at discharge and 1- year mortality in hospitalised patients with acute decompensated heart failure: an observational study
}

\author{
Yuji Nishimoto, ${ }^{1}$ Takao Kato (D) , ${ }^{2}$ Takeshi Morimoto (D) , ${ }^{3}$ Hidenori Yaku, ${ }^{2}$ \\ Yasutaka Inuzuka, ${ }^{4}$ Yodo Tamaki, ${ }^{5}$ Erika Yamamoto, ${ }^{2}$ Yusuke Yoshikawa, ${ }^{2}$ \\ Takeshi Kitai, ${ }^{6}$ Ryoji Taniguchi, ${ }^{1}$ Moritake Iguchi (10, , ${ }^{7}$ Masashi Kato, ${ }^{8}$ \\ Mamoru Takahashi, ${ }^{9}$ Toshikazu Jinnai, ${ }^{10}$ Tomoyuki Ikeda, ${ }^{11}$ Kazuya Nagao (D) , ${ }^{12}$ \\ Takafumi Kawai, ${ }^{13}$ Akihiro Komasa, ${ }^{14}$ Ryusuke Nishikawa, ${ }^{15}$ Yuichi Kawase, ${ }^{16}$ \\ Takashi Morinaga, ${ }^{17}$ Kanae Su, ${ }^{18}$ Mitsunori Kawato, ${ }^{19}$ Yuta Seko, ${ }^{20}$ Moriaki Inoko, ${ }^{20}$ \\ Mamoru Toyofuku, ${ }^{18}$ Yutaka Furukawa, ${ }^{6}$ Yoshihisa Nakagawa, ${ }^{5}$ Kenji Ando, ${ }^{17}$ \\ Kazushige Kadota, ${ }^{16}$ Satoshi Shizuta (D) , ${ }^{2}$ Koh Ono, ${ }^{2}$ Koichiro Kuwahara, ${ }^{21}$ \\ Neiko Ozasa, ${ }^{2}$ Yukihito Sato, ${ }^{1}$ Takeshi Kimura (i) ${ }^{2}$
}

To cite: Nishimoto Y, Kato T, Morimoto T, et al. C-reactive protein at discharge and 1year mortality in hospitalised patients with acute decompensated heart failure: an observational study. BMJ Open 2020;10:e041068. doi:10.1136/ bmjopen-2020-041068

- Prepublication history and additional materials for this paper is available online. To view these files, please visit the journal online (http://dx.doi. org/10.1136/bmjopen-2020041068).

Received 29 May 2020 Revised 15 December 2020 Accepted 15 December 2020

Check for updates

(c) Author(s) (or their employer(s)) 2020. Re-use permitted under CC BY-NC. No commercial re-use. See rights and permissions. Published by BMJ.

For numbered affiliations see end of article.

Correspondence to

Dr Takao Kato;

tkato75@kuhp.kyoto-u.ac.jp

\section{ABSTRACT}

Objectives To examine the association of a high Creactive protein (CRP) level at discharge from an acute decompensated heart failure (ADHF) hospitalisation with the 1-year clinical outcomes.

Design A post-hoc subanalysis of a prospective cohort study of patients hospitalised for ADHF (using the Kyoto Congestive Heart Failure (KCHF) registry) between October 2014 and March 2016 with a 1-year follow-up.

Setting A physician-initiated multicentre registry enrolled consecutive hospitalised patients with ADHF for the first time at 19 secondary and tertiary hospitals in Japan.

Participants Among the 4056 patients enrolled in the KCHF registry, the present study population consisted of 2618 patients with an available CRP value both on admission and at discharge and post-discharge clinical follow-up data. We divided the patients into two groups, those with a high CRP level ( $>10 \mathrm{mg} / \mathrm{L}$ ) and those with a low CRP level $(\leq 10 \mathrm{mg} / \mathrm{L})$ at discharge from the index hospitalisation.

Primary and secondary outcome measures The primary outcome measure was all-cause death after discharge from the index hospitalisation. The secondary outcome measures were heart failure hospitalisations, cardiovascular death and non-cardiovascular death.

Results The high CRP group and low CRP group included 622 patients (24\%) and 1996 patients $(76 \%)$, respectively. During a median follow-up period of 468 days, the cumulative 1-year incidence of the primary outcome was significantly higher in the high CRP group than low CRP group $(24.1 \%$ vs $13.9 \%$, log-rank $p<0.001)$. Even after a multivariable analysis, the excess mortality risk in the high CRP group relative to the low CRP group remained significant ( $\mathrm{HR}, 1.43 ; 95 \% \mathrm{Cl}, 1.19$ to $1.71 ; \mathrm{p}<0.001)$. The excess mortality risk was consistent regardless of the clinically relevant subgroup factors.

\section{Strengths and limitations of this study}

This study was a large-scale, contemporary, multicentre, observational study clarifying the association of a high C-reactive protein (CRP) level at discharge from an acute decompensated heart failure (ADHF) hospitalisation with the 1-year clinical outcomes.

- The data for this study were prospectively collected from consecutive patients who were hospitalised due to ADHF in the real-world clinical practice in Japan.

- This study examined whether a high CRP level (>10 mg/L) at discharge from an ADHF hospitalisation was associated with an excess mortality risk at 1 year.

We could not fully address the effects of chronic inflammatory diseases such as autoimmune disease and malignancy on the clinical outcomes.

Conclusions A high CRP level ( $>10 \mathrm{mg} / \mathrm{L})$ at discharge from an ADHF hospitalisation was associated with an excess mortality risk at 1 year.

Trial registration details $\mathrm{https} / / / \mathrm{clinicaltrials.gov/ct2/}$ show/NCT02334891 (NCT02334891) https://upload. umin.ac.jp/cgi-open-bin/ctr_e/ctr_view.cgi?recptno= R000017241 (UMIN000015238).

\section{INTRODUCTION}

The post-discharge mortality in patients with acute decompensated heart failure (ADHF) remains high and have not significantly improved over the past decade. ${ }^{1}$ The previous studies reported that the cumulative 1-year incidence of all-cause death in hospitalised patients with ADHF was approximately $20 \%$, 
which was substantially higher than that in patients with chronic heart failure who remained free from ADHF hospitalisations. ${ }^{23}$ Thus, identification of patients with a high mortality risk is clinically relevant in hospitalised patients with ADHF.

The C-reactive protein (CRP) is a product of the liver regulated by cytokines, principally interleukin 6 (IL-6) and tumour necrosis factor- $\alpha$ (TNF- $\alpha$ ) and activates the classical complement pathway and opsonises phagocytic ligands. ${ }^{45}$ The CRP has been established as a prognostic predictor of adverse events in patients with chronic heart failure ${ }^{6-8}$ but has not been adequately established in patients with ADHF. Some previous studies have shown that high CRP levels on admission due to ADHF are associated with a high mortality after discharge $;^{9-11}$ however, those results were affected by acute inflammatory responses such as a concomitant infection and acute coronary syndrome (ACS). On the other hand, the CRP level at discharge from an ADHF hospitalisation might reflect a chronic inflammatory response and could be a more relevant prognostic predictor in hospitalised patients with ADHF. Thus, we aimed to examine the association of a high CRP level at discharge from an ADHF hospitalisation with the 1-year clinical outcomes using a large contemporary all-comer study of patients with ADHF hospitalisations in Japan.

\section{METHODS}

\section{Study design, setting and population}

The KCHF (Kyoto Congestive Heart Failure) registry was a physician-initiated, prospective, observational, multicentre cohort study enrolling consecutive patients who were hospitalised due to ADHF for the first time between October 2014 and March 2016, including patients with previous heart failure hospitalisations before October 2014. The participating centres were 19 secondary and tertiary hospitals, including rural and urban, as well as large and small institutions, in Japan. The design and patient enrolment in the KCHF registry were previously reported in detail. ${ }^{13}$ Briefly, we enrolled consecutive patients with ADHF as defined by the modified Framingham criteria, who were admitted to the participating hospitals and underwent heart failure-specific treatment requiring intravenous drugs within 24 hours after presenting to the hospitals. One-year clinical follow-up data were collected in October 2017. The attending physicians or research assistants at each participating hospital collected the clinical event data, including deaths and heart failure hospitalisations, during the follow-up from the hospital medical records or patients, their relatives or their referring physicians by phone and/or mailed questions.

Among the 4056 patients enrolled in the KCHF registry, 271 died during the index hospitalisation (figure 1). We excluded patients without data after the index hospitalisation $(\mathrm{n}=57)$, patients with missing CRP values on admission and/or at discharge $(n=413)$, and those with a fever

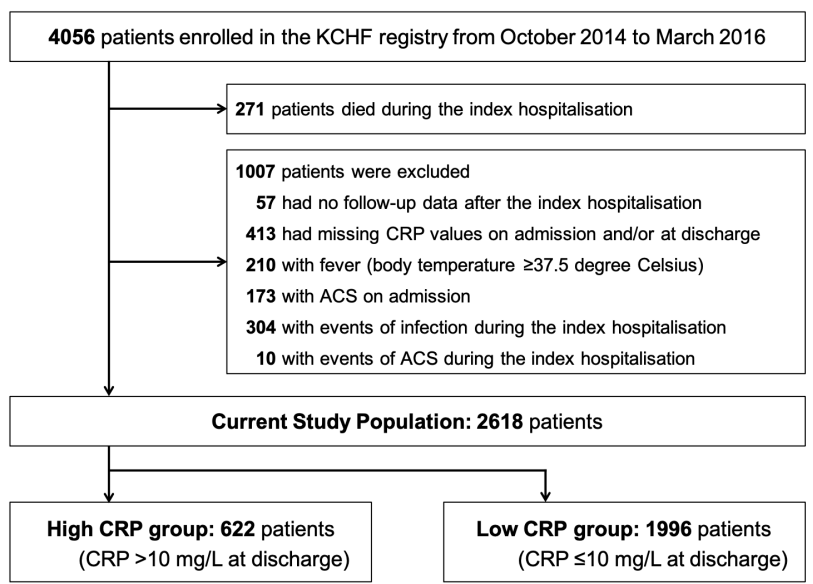

Figure 1 Study flowchart. ACS, acute coronary syndrome; CRP, C-reactive protein; KCHF, Kyoto Congestive Heart Failure.

$(n=210), \operatorname{ACS}(n=173)$, infectious events during the index hospitalisation $(\mathrm{n}=304)$ and ACS events during the index hospitalisation $(n=10)$. Finally, the present study population consisted of 2618 patients, including 1658 patients $(63 \%)$ with first-ever heart failure hospitalisations, with an available CRP value both on admission and at discharge and post-discharge clinical follow-up data. According to the previously reported cut-off values, ${ }^{9}{ }^{14}$ we divided the patients into two groups, those with a high CRP level $(>10 \mathrm{mg} / \mathrm{L})$ and those with a low CRP level $(\leq 10 \mathrm{mg} / \mathrm{L})$ at discharge from the index hospitalisation (figure 1).

\section{Ethics}

The investigation conformed to the principles outlined in the Declaration of Helsinki. The study protocol was approved by the ethical committees at Kyoto University Hospital (local identifier: E2311) and at each participating hospital (online supplemental eAppendix 1). A waiver of written informed consent from each patient was approved, because it met the conditions included in the Japanese Ethical Guidelines for Epidemiological Studies. ${ }^{1213}$ No patients refused to participate in the study when contacted for follow-up.

\section{Patient and public involvement}

This research was done without patient involvement. Patients were not invited to comment on the study design and were not consulted to develop patient-relevant outcomes or to interpret the results. Patients were not invited to contribute to the writing or editing of this document for readability or accuracy.

\section{Definitions}

The CRP values were measured on admission and at discharge in each hospital using a normal-sensitivity or high-sensitivity assay. The CRP measurement in each hospital was standardised by the common reference interval developed by the Committee on Common Reference Intervals of the Japan Society of Clinical Chemistry. ${ }^{15}$ 
Fever was defined as a body temperature of $>37.5$ degree Celsius. Infection included viral infections, bacterial pneumonia, urinary tract infections, biliary tract infections, sepsis, unknown foci of the infections and other infections (online supplemental eTable 1). Heart failure was classified according to the baseline left ventricular ejection fraction (LVEF) as that with a reduced LVEF $(<40 \%)$ or preserved LVEF $(\geq 40 \%)$. Anaemia was diagnosed if the value of the haemoglobin was $<13 \mathrm{~g} / \mathrm{dL}$ for men and $<12 \mathrm{~g} / \mathrm{dL}$ for women. The detailed definitions of the other patient characteristics are described in online supplemental eAppendix 2. The missing values are presented in online supplemental eTable 2.

The primary outcome measure in the present study was all-cause death after discharge from the index hospitalisation. Other outcome measures included heart failure hospitalisations, cardiovascular death and noncardiovascular death. The causes of death were classified according to the VARC (Valve Academic Research Consortium) definitions, ${ }^{16}$ and were adjudicated by a clinical event committee. ${ }^{12} 1317$ Death was regarded as cardiovascular in origin unless obvious non-cardiovascular causes could be identified. Cardiovascular death included death related to heart failure, acute myocardial infarctions, fatal ventricular arrhythmias, sudden cardiac death (SCD), other cardiac death, strokes, intracranial haemorrhages and other vascular death. ${ }^{17}$ SCD was defined as unexplained death of a previously stable patient, including fatal ventricular arrhythmias and cardiac arrest. Noncardiovascular death included malignancy, infections, renal failure, liver failure, respiratory failure, bleeding and other causes. ${ }^{17}$ Heart failure hospitalisations were due to worsening heart failure, requiring intravenous drug therapy. ${ }^{12}$

\section{Statistical analysis}

Categorical variables are expressed as numbers and percentages, and continuous variables are expressed as the mean with the SD or median with the IQR based on their distribution. As for the patient characteristics, the categorical variables were compared using the $\chi^{2}$ test when appropriate; otherwise, the Fisher's exact test was used. Continuous variables were compared using the Student's t-test or Wilcoxon rank-sum test based on their distribution. The baseline characteristics and clinical outcomes including all-cause death, heart failure hospitalisations, cardiovascular death and noncardiovascular death were compared between the high CRP and low CRP groups at discharge. We regarded the date of discharge as time zero for the clinical follow-up. The 1-year clinical follow-up was regarded as completed with an allowance of 1 month. Cumulative incidences were estimated by the Kaplan-Meier method and differences were assessed with the log-rank test. We constructed multivariable Cox proportional hazard models to estimate the risk of the high CRP group relative to the low CRP group, with the results expressed as the HRs and 95\% CIs. We included the following 25 clinically relevant risk-adjusting variables into the model: demographical variables (age $\geq 80$ years, sex and body mass index (BMI) $\leq 22 \mathrm{~kg} / \mathrm{m}^{2}$ ), variables related to heart failure (previous heart failure hospitalisation and LVEF $<40 \%$ by echocardiography), variables related to comorbidities (atrial fibrillation or flutter, hypertension, diabetes mellitus, previous myocardial infarction, previous stroke, current smoking and chronic lung disease), living status (living alone and ambulatory), vital signs at presentation (systolic blood pressure $<90 \mathrm{~mm} \mathrm{Hg}$ and heart rate $<60 \mathrm{bpm}$ ), laboratory tests on admission (estimated glomerular filtration rate (eGFR) $<30 \mathrm{~mL} / \mathrm{min} / 1.73 \mathrm{~m}^{2}$, albumin $<3.0 \mathrm{~g} / \mathrm{dL}$, sodium $<135 \mathrm{mEq} / \mathrm{L}$ and anaemia) and medications at discharge (ACE inhibitors (ACEIs) or angiotensin II receptor blockers (ARBs), $\beta$-blockers, loop diuretics and tolvaptan) consistent with the previous reports, ${ }^{18-20}$ and history of malignancy as an additional variable into the model, as indicated in table 1 . The continuous variables were dichotomised by clinically meaningful reference values or median values. To account for the competing risk of all-cause death, we used the Fine and Gray's method to estimate the risk for heart failure hospitalisations in the high CRP group relative to the low CRP group. As sensitivity analyses, we constructed three additional multivariable Cox proportional hazard models. First, we set a lower cut-off value for the CRP, and estimated the risk of the group with a CRP level $>3 \mathrm{mg} / \mathrm{L}$ relative to the group with a CRP level $\leq 3 \mathrm{mg} / \mathrm{L}$ for the primary outcome measure. Second, we excluded those patients with very high CRP levels $(>50 \mathrm{mg} / \mathrm{L})$ and estimated the risk of the high CRP group (CRP $>10 \mathrm{mg} / \mathrm{L}$ ) relative to the low CRP group (CRP $\leq 10 \mathrm{mg} / \mathrm{L}$ ) for the primary outcome measure. Finally, based on the quartile of the CRP levels at discharge, we divided the entire cohort into four groups: Quartile 1 $(\leq 1.3 \mathrm{mg} / \mathrm{L})$, Quartile 2 (1.4 to $3.5 \mathrm{mg} / \mathrm{L})$, Quartile $3(3.6$ to $9.5 \mathrm{mg} / \mathrm{L})$ and Quartile $4(\geq 9.6 \mathrm{mg} / \mathrm{L})$. We compared the clinical outcomes across the CRP quartiles, and estimated the risk of Quartile 2, Quartile 3 and Quartile 4, relative to Quartile 1 for the primary outcome measure. In the post-hoc subgroup analysis, we evaluated the interaction between those subgroup factors such as the age, coronary artery disease and LVEF at baseline, and the risk of the high CRP group relative to the low CRP group for the primary outcome measure in the Cox models. All statistical analyses were performed with EZR software (Saitama Medical Center, Jichi Medical University, Saitama, Japan) or JMP V.14.0.0 (SAS Institute Inc, Cary, North Carolina, USA). Two tailed $\mathrm{p}$ values of $<0.05$ were considered statistically significant.

\section{Data sharing statement}

All data relevant to the study are included in the article or uploaded as supplementary information. No additional data available.

\section{RESULTS}

\section{Patient characteristics}

In the present study population, the median CRP level at discharge in the entire study population was 3.6 (IQR, 1.4 to 9.6$) \mathrm{mg} / \mathrm{L}$ (figure 2). The high CRP group (CRP $>10 \mathrm{mg} / \mathrm{L})$ and low CRP group $(\mathrm{CRP} \leq 10 \mathrm{mg} / \mathrm{L})$ included 622 patients (24\%) and 1996 patients $(76 \%)$, respectively 
Table 1 Patient characteristics

\begin{tabular}{|c|c|c|c|c|}
\hline & Total $(n=2618)$ & $\begin{array}{l}\text { High CRP group } \\
\text { CRP }>10 \mathrm{mg} / \mathrm{L}(\mathrm{n}=622)\end{array}$ & $\begin{array}{l}\text { Low CRP group } \\
\text { CRP } \leq 10 \mathrm{mg} / \mathrm{L} \\
(\mathrm{n}=1996)\end{array}$ & $P$ value \\
\hline $\mathrm{CRP}$ at discharge, $\mathrm{mg} / \mathrm{L}$ & 3.6 (1.4 to 9.6$)$ & 19.6 (13.4 to 31.9$)$ & 2.1 (1.0 to 4.9$)$ & $<0.001$ \\
\hline Lowest and highest decile, mg/L & 0.6 to 22.0 & 11.3 to 55.7 & 0.5 to 7.4 & \\
\hline Delta CRP†, mg/L & $7.5 \pm 31.1$ & $-0.05 \pm 44.9$ & $9.9 \pm 24.8$ & $<0.001$ \\
\hline Length of hospital stay, days & 15 (11 to 22$)$ & 15 (10 to 23$)$ & 15 (11 to 22$)$ & 0.29 \\
\hline \multicolumn{5}{|l|}{ Clinical characteristics } \\
\hline Age, years & $77.6 \pm 12.1$ & $79.4 \pm 10.9$ & $77.0 \pm 12.3$ & $<0.001$ \\
\hline$\geq 80$ years $^{*}$ & $1354(52 \%)$ & $354(57 \%)$ & $1000(50 \%)$ & 0.003 \\
\hline Women* & $1186(45 \%)$ & 240 (39\%) & $946(47 \%)$ & $<0.001$ \\
\hline $\mathrm{BMI}, \mathrm{kg} / \mathrm{m}^{2}$ & $22.9 \pm 4.5$ & $22.8 \pm 4.4$ & $22.9 \pm 4.6$ & 0.46 \\
\hline$\leq 22 \mathrm{~kg} / \mathrm{m}^{2 *}$ & $1161(46 \%)$ & $263(45 \%)$ & $898(47 \%)$ & 0.55 \\
\hline Aetiology & & & & $<0.001$ \\
\hline Coronary artery disease excluding ACS & 728 (28\%) & $194(31 \%)$ & $534(27 \%)$ & \\
\hline Hypertensive heart disease & $698(27 \%)$ & $163(26 \%)$ & $535(27 \%)$ & \\
\hline Cardiomyopathy & $442(17 \%)$ & $76(12 \%)$ & $366(18 \%)$ & \\
\hline Valvular heart disease & $532(20 \%)$ & $130(21 \%)$ & $402(20 \%)$ & \\
\hline Other heart disease & $65(2.5 \%)$ & $24(3.9 \%)$ & $41(2.1 \%)$ & \\
\hline \multicolumn{5}{|l|}{ Medical history } \\
\hline Previous heart failure hospitalisation* & $960(37 \%)$ & $215(35 \%)$ & $745(38 \%)$ & 0.25 \\
\hline Atrial fibrillation or flutter ${ }^{\star}$ & $1162(44 \%)$ & $251(40 \%)$ & $911(46 \%)$ & 0.02 \\
\hline Hypertension* & $1891(72 \%)$ & $463(74 \%)$ & $1428(72 \%)$ & 0.16 \\
\hline Diabetes mellitus* & $936(36 \%)$ & $228(37 \%)$ & $708(35 \%)$ & 0.59 \\
\hline Dyslipidaemia & $1010(39 \%)$ & $244(39 \%)$ & 766 (38\%) & 0.70 \\
\hline Previous myocardial infarction* & $584(22 \%)$ & $155(25 \%)$ & $429(21 \%)$ & 0.07 \\
\hline Previous stroke* & $414(16 \%)$ & $110(18 \%)$ & $304(15 \%)$ & 0.14 \\
\hline Previous $\mathrm{PCl}$ or $\mathrm{CABG}$ & $682(26 \%)$ & $187(30 \%)$ & $495(25 \%)$ & 0.009 \\
\hline Current smoking* & $324(13 \%)$ & $80(13 \%)$ & $244(12 \%)$ & 0.60 \\
\hline Chronic kidney disease & $1153(44 \%)$ & 302 (49\%) & $851(43 \%)$ & 0.009 \\
\hline Chronic lung disease ${ }^{\star}$ & $345(13 \%)$ & $105(17 \%)$ & $240(12 \%)$ & 0.002 \\
\hline Malignancy* & $385(15 \%)$ & $93(15 \%)$ & $292(15 \%)$ & 0.84 \\
\hline Cognitive dysfunction & $468(18 \%)$ & $132(21 \%)$ & $336(17 \%)$ & 0.01 \\
\hline \multicolumn{5}{|l|}{ Social backgrounds } \\
\hline Poor medical adherence & $458(17 \%)$ & $108(17 \%)$ & $350(18 \%)$ & 0.92 \\
\hline Living alone* & $563(22 \%)$ & $118(19 \%)$ & $445(22 \%)$ & 0.08 \\
\hline With occupation & $343(13 \%)$ & $72(12 \%)$ & $271(14 \%)$ & 0.20 \\
\hline Public financial assistance & $150(5.7 \%)$ & $33(5.3 \%)$ & $117(5.9 \%)$ & 0.60 \\
\hline Daily life activities & & & & $<0.001$ \\
\hline Ambulatory* & $2083(80 \%)$ & $457(74 \%)$ & $1626(82 \%)$ & \\
\hline Use of wheelchair, outdoor only & $189(7.3 \%)$ & $56(9.1 \%)$ & $133(6.7 \%)$ & \\
\hline Use of wheelchair, outdoor and indoor & $232(8.9 \%)$ & $78(13 \%)$ & $154(7.8 \%)$ & \\
\hline Bedridden & $90(3.5 \%)$ & $25(4.1 \%)$ & $65(3.3 \%)$ & \\
\hline \multicolumn{5}{|l|}{ Vital signs at presentation } \\
\hline \multicolumn{5}{|l|}{$\mathrm{BP}, \mathrm{mm} \mathrm{Hg}$} \\
\hline Systolic BP & $149 \pm 35$ & $150 \pm 36$ & $148 \pm 35$ & 0.37 \\
\hline
\end{tabular}


Table 1 Continued

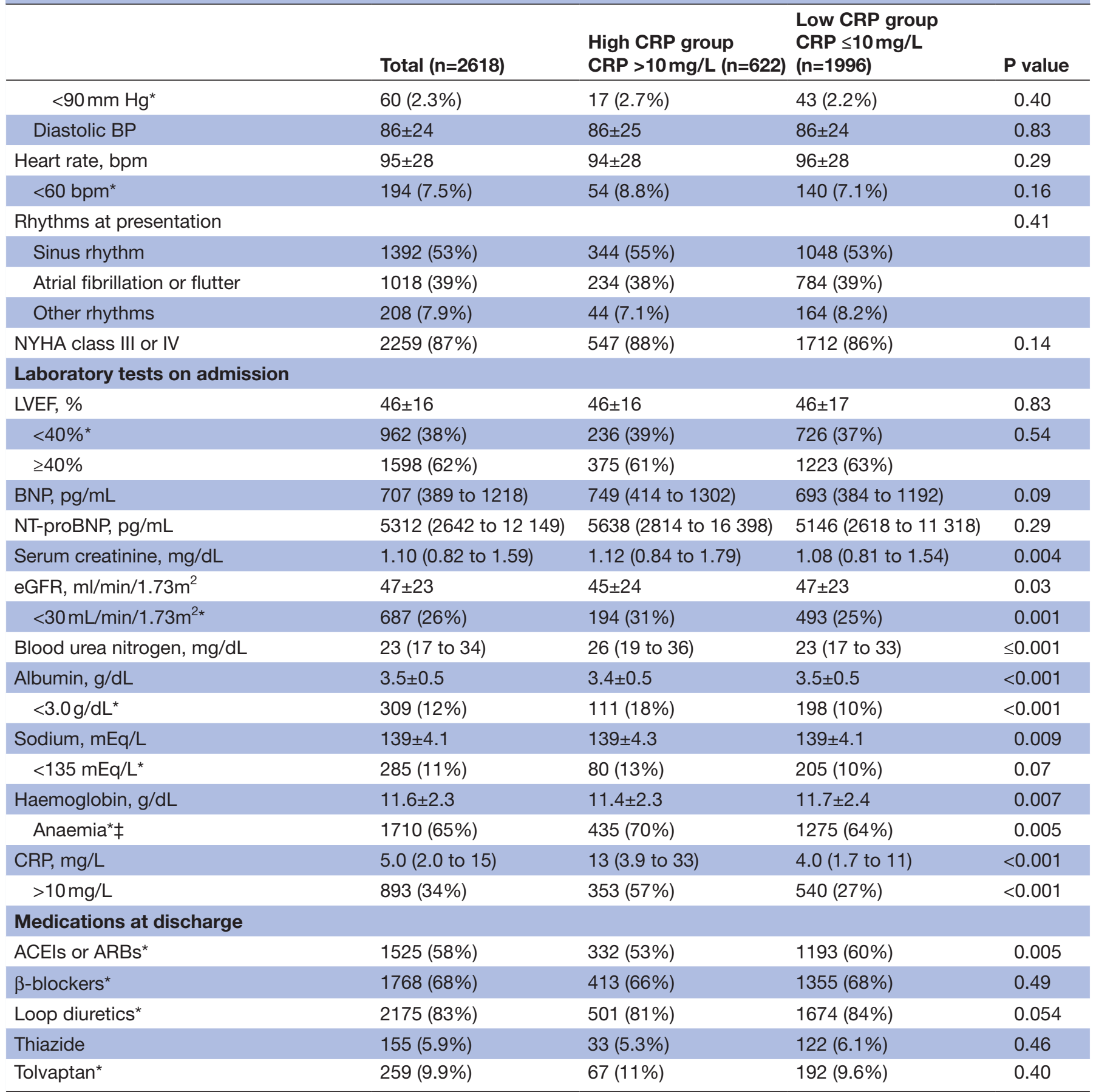

Categorical variables are presented as numbers and percentages. Continuous variables are presented as the mean $\pm \mathrm{SD}$, or the median (IQR) based on their distributions. Categorical variables were compared with the $\chi^{2}$ test. Continuous variables were compared using the Student's t-test or Wilcoxon rank-sum test based on their distribution.

Missing values were presented in online supplemental eTable 2.

${ }^{*}$ Risk-adjusting variables selected for multivariable Cox proportional hazard models.

†Delta CRP levels were calculated according to the following equation: (the CRP levels on admission) - (the CRP levels at discharge). $\ddagger$ Anaemia was defined by the WHO criteria (haemoglobin $<12.0 \mathrm{~g} / \mathrm{dL}$ in women and $13.0 \mathrm{~g} / \mathrm{dL}$ in men).

ACEI, ACE inhibitor; ACS, acute coronary syndrome; ARB, angiotensin II receptor blocker; BMI, body mass index; BNP, brain-type natriuretic peptide; BP, blood pressure; bpm, beat per minute; CABG, coronary artery bypass grafting; CRP, C-reactive protein; eGFR, estimated glomerular filtration rate; LVEF, left ventricular ejection fraction; NT-proBNP, N-terminal-proBNP; NYHA, New York Heart Association; PCI, percutaneous coronary intervention. 


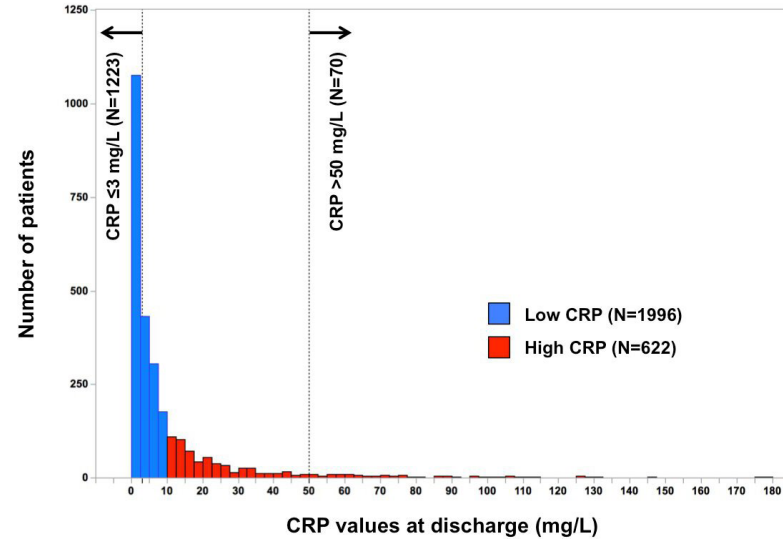

Figure 2 Distribution of the CRP values at discharge. CRP, C-reactive protein.

(figure 1). The baseline characteristics were significantly different in several aspects between the high and low CRP groups (table 1). Patients in the high CRP group were older, less frequently women, and more frequently had coronary artery disease excluding ACS, previous percutaneous coronary intervention or coronary artery bypass grafting, chronic kidney disease, chronic lung disease, dementia, high blood urea nitrogen levels, low albumin levels, hyponatraemia and anaemia than those in the low CRP group. On the other hand, the patients in the low CRP group more frequently had an ambulatory status and the use of ACEIs or ARBs at discharge (table 1).

\section{Clinical outcomes}

The median length of the follow-up was 468 (IQR, 362 to 637 ) days, with a $95.8 \%$ follow-up rate at 1 year. The cumulative 1-year incidence of the primary outcome measure (all-cause death after discharge) was significantly higher in the high CRP group than low CRP group $(24.1 \%$ vs $13.9 \%, \mathrm{p}<0.001)$ (figure $3 \mathrm{~A})$. After a multivariable analysis, the excess risk of the high CRP group relative to the low CRP group remained significant for all-cause death (adjusted HR, 1.43; 95\% CI, 1.19 to $1.71 ; \mathrm{p}<0.001$ ) (table 2 ). The excess adjusted risks of the high CRP group relative to the low CRP group were also significant for cardiovascular death and noncardiovascular death (adjusted HR, 1.31; 95\% CI, 1.03 to 1.65; $\mathrm{p}=0.03$, and adjusted HR, 1.60; 95\% CI, 1.21 to 2.12; $\mathrm{p}<0.001$, respectively) (table 2 ). The cumulative 1-year incidence of a heart failure hospitalisation after discharge was not different between the high and low CRP groups (27.5\% vs $24.6 \%, \mathrm{p}=0.23$ ) (figure $3 \mathrm{~B}$ ). Even after a multivariable analysis, the risk of the high CRP group relative to the low CRP group remained insignificant for a heart failure hospitalisation (adjusted HR, 1.04; 95\% CI, 0.88 to 1.25; $\mathrm{p}=0.63$ ) (table 2). Even after taking into account the competing risk of all-cause death, the cumulative 1-year incidence of a heart failure hospitalisation was not different between the high and low CRP groups (25.0\% vs $23.8 \%, \mathrm{p}=0.73$ ) (online supplemental eFigure 1), and the adjusted risk of the high CRP group relative to the low CRP group remained insignificant for a heart failure hospitalisation (HR, 0.96 ; 95\% CI, 0.80 to 1.16 ; $\mathrm{p}=0.69$ ) (online supplemental eTable 3 ). The details of the causes of death in the high and low CRP groups are presented in table 3 .

\section{Sensitivity analysis}

Even when we divided the patients into two groups of patients according to a CRP level of $3 \mathrm{mg} / \mathrm{L}$ (online supplemental eTable 4), the excess crude and adjusted risks in the CRP $>3 \mathrm{mg} / \mathrm{L}$ group relative to the $\mathrm{CRP} \leq 3 \mathrm{mg} / \mathrm{L}$ group for the primary outcome measure remained significant (adjusted HR, 1.47; 95\% CI, 1.23 to $1.75 ; \mathrm{p}<0.001$ ) (online supplemental eTable 5). In addition, even after excluding the patients with a CRP level $>50 \mathrm{mg} / \mathrm{L}(\mathrm{n}=2548$, online
A
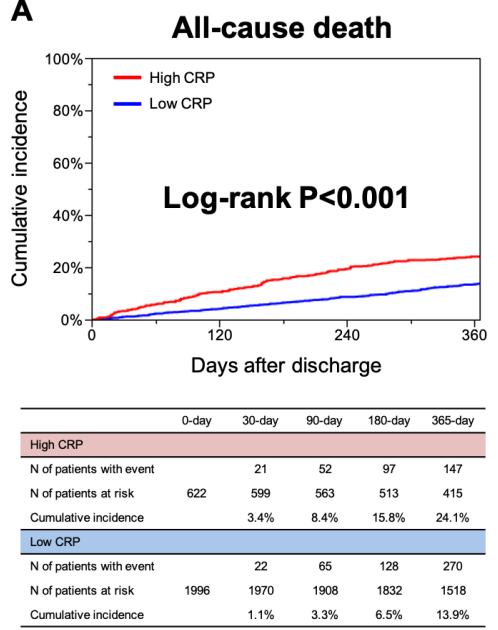

B

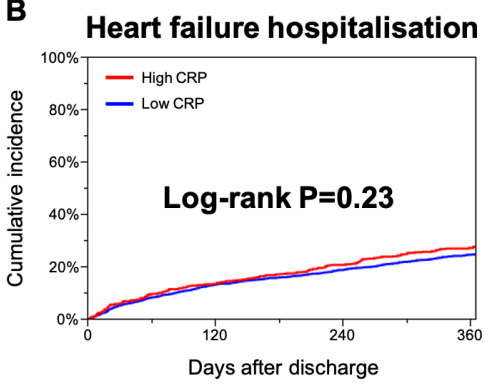

\begin{tabular}{lccccc}
\hline & 0-day & 30-day & 90 -day & 180 -day & 365-day \\
\hline High CRP & & & & & \\
\hline N of patients with event & & 36 & 72 & 101 & 153 \\
N of patients at risk & 622 & 570 & 507 & 446 & 322 \\
Cumulative incidence & & $5.9 \%$ & $11.9 \%$ & $17.2 \%$ & $27.5 \%$ \\
\hline Low CRP & & & & & \\
\hline N of patients with event & & 101 & 209 & 308 & 468 \\
N of patients at risk & 1996 & 1878 & 1732 & 1589 & 1201 \\
Cumulative incidence & & $5.1 \%$ & $10.6 \%$ & $15.8 \%$ & $24.6 \%$ \\
\hline
\end{tabular}

Figure 3 The Kaplan-Meier curves (A) for all-cause death after discharge compared between the high CRP group (CRP $>10 \mathrm{mg} / \mathrm{L}$ at discharge) versus low CRP group (CRP $\leq 10 \mathrm{mg} / \mathrm{L}$ at discharge), and (B) for heart failure hospitalisations after discharge compared between the high CRP group (CRP $>10 \mathrm{mg} / \mathrm{L}$ at discharge) versus low CRP group (CRP $\leq 10 \mathrm{mg} / \mathrm{L}$ at discharge). CRP, C-reactive protein. 
Table 2 Clinical outcomes

\begin{tabular}{|c|c|c|c|c|c|c|}
\hline & $\begin{array}{l}\text { High CRP group } \\
\text { CRP }>10 \mathrm{mg} / \mathrm{L}(\mathrm{n}=622) \\
\mathrm{N} \text { of patients with event } \\
\text { (cumulative 1-year } \\
\text { incidence) }\end{array}$ & $\begin{array}{l}\text { Low CRP group } \\
\text { CRP } \leq 10 \mathrm{mg} / \mathrm{L}(\mathrm{n}=1996) \\
\mathrm{N} \text { of patients with event } \\
\text { (cumulative 1-year } \\
\text { incidence) }\end{array}$ & Crude HR $(95 \% \mathrm{Cl})$ & $P$ value & Adjusted HR (95\% Cl) & $P$ value \\
\hline All-cause death & $190(24.1 \%)$ & $391(13.9 \%)$ & 1.75 (1.47 to 2.07 ) & $<0.001$ & $1.43(1.19$ to 1.71$)$ & $<0.001$ \\
\hline $\begin{array}{l}\text { Cardiovascular } \\
\text { death }\end{array}$ & $107(14.4 \%)$ & 247 (9.0\%) & 1.56 (1.24 to 1.95$)$ & $<0.001$ & 1.31 (1.03 to 1.65$)$ & 0.03 \\
\hline $\begin{array}{l}\text { Heart failure } \\
\text { hospitalisation }\end{array}$ & $170(27.5 \%)$ & 537 (24.6\%) & 1.11 (0.93 to 1.32$)$ & 0.24 & 1.04 (0.88 to 1.25$)$ & 0.63 \\
\hline
\end{tabular}

Number of patients with event was counted throughout the entire follow-up period because we used the Cox proportional hazard models which provided the HRs between the high and low CRP groups over the entire study duration, while the cumulative incidence was estimated at 1 year. The crude and adjusted HRs and $95 \%$ Cls of the high CRP group for the clinical outcome measures were estimated by the Cox proportional hazard models using the low CRP group as the reference. To adjust for potential confounders, we selected 25 clinically relevant confounders as indicated in table 1.

CRP, C-reactive protein.;

supplemental eTable 6), the excess crude and adjusted risks in the high CRP group (CRP $>10 \mathrm{mg} / \mathrm{L})$ relative to the low CRP group (CRP $\leq 10 \mathrm{mg} / \mathrm{L})$ for the primary outcome measure remained significant (adjusted HR, 1.37; 95\% CI, 1.14 to $1.65 ; \mathrm{p}=0.001$ ) (online supplemental eTable 7 ). When we divided the entire cohort into four groups based on the quartile of the CRP levels at discharge, the cumulative 1-year incidence of the primary outcome measure increased incrementally from Quartile 1 to Quartile 4 (Quartile 1: $10.5 \%$, Quartile 2: 12.9\%, Quartile 3: 17.8\% and Quartile 4: 23.7\%,

Table 3 Causes of death compared between the high CRP group versus low CRP group during entire follow-up period

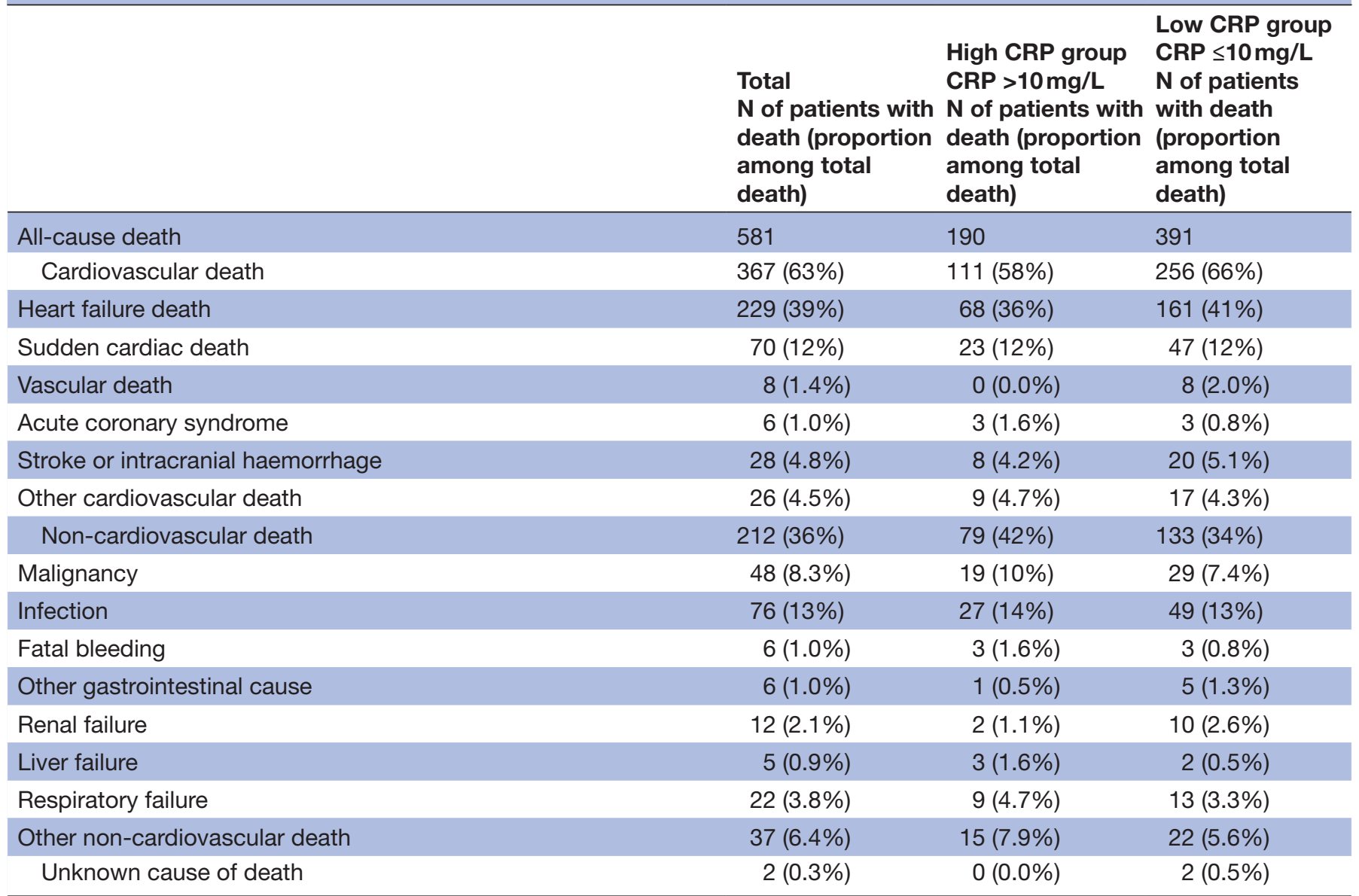

Number of patients with death was counted throughout the entire follow-up period because we used the Cox proportional hazard models, which provided the HRs between the high and low CRP groups over the entire study duration.

CRP, C-reactive protein. 


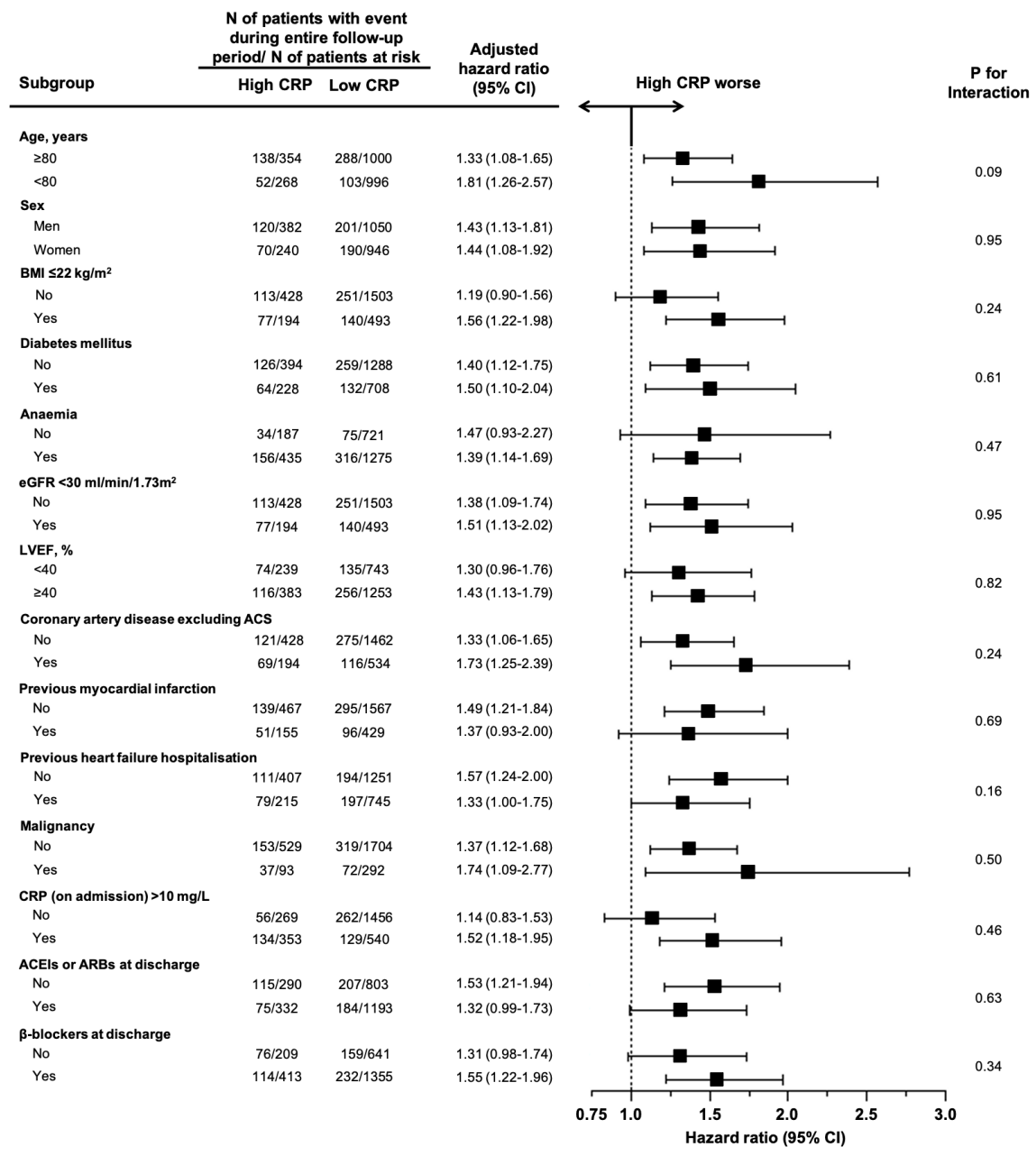

Figure 4 Subgroup analysis for the associations of the high CRP group relative to the low CRP group with all-cause deaths after discharge. Anaemia was defined by the WHO criteria (haemoglobin $<12.0 \mathrm{~g} / \mathrm{dL}$ in women and $13.0 \mathrm{~g} / \mathrm{dL}$ in men). ACEI, angiotensin converting enzyme inhibitor; ACS, acute coronary syndrome; ARB, angiotensin II receptor blocker; BMI, body mass index; CRP, C-reactive protein; eGFR, estimated glomerular filtration rate; LVEF, left ventricularejection fraction.

$\mathrm{p}<0.001$ ) (online supplemental eFigure 2). Even after a multivariable analysis, the excess risk of Quartile 4 relative to Quartile 1 remained significant for the primary outcome measure (adjusted HR, 1.95; 95\% CI, 1.51 to 2.52; $\mathrm{p}<0.001$ ) (online supplemental eTable 8). The cumulative 1-year incidence of heart failure hospitalisations after discharge was not different across the CRP quartiles $(23.4 \%, 23.1 \%, 27.4 \%$ and $27.3 \%, \mathrm{p}=0.25$ ) (online supplemental eFigure 2). Even after the multivariable analysis, the risk of Quartile 4 relative to Quartile 1 remained insignificant for heart failure hospitalisations (adjusted HR, 1.04; 95\% CI, 0.84 to 1.29 ; $\mathrm{p}=0.71$ ) (online supplemental eTable 8).

\section{Subgroup analysis}

We conducted subgroup analyses stratified by the age, sex, BMI, diabetes mellitus, anaemia, renal dysfunction, LVEF, coronary artery disease excluding ACS, previous myocardial infarction, previous heart failure hospitalisation, malignancy, elevated CRP level $(>10 \mathrm{mg} / \mathrm{L})$ on admission, use of ACEIs or ARBs at discharge and use of $\beta$-blockers at discharge (figure 4). The CRP level at discharge was higher in the elderly patients ( $\geq 80$ years), men, and patients with anaemia, renal dysfunction (eGFR $<30 \mathrm{~mL} / \mathrm{min} / 1.73 \mathrm{~m}^{2}$ ), coronary artery disease excluding ACS, previous heart failure hospitalisations, elevated CRP levels on admission and the use of the ACEIs or ARBs at discharge (online supplemental eTable 9). The CRP level at discharge was not different regardless of a reduced or preserved LVEF (online supplemental eTable 9). An excess adjusted risk in the high CRP group relative to the low CRP group for the primary outcome measure was consistently seen across all the subgroups (figure 4 ). There were no significant interactions between the subgroup factors and effect of the high CRP group relative to the low CRP group for the primary outcome measure (figure 4).

\section{DISCUSSION}

The main findings of the present study were as follows: (1) the high CRP level $(>10 \mathrm{mg} / \mathrm{L})$ at discharge from the index hospitalisation for ADHF was associated with an excess adjusted risk for all-cause death at 1 year; (2) the 
excess mortality risk in the high CRP group relative to the low CRP group was consistent in all the clinically relevant subgroups; and (3) there was no significant excess risk for heart failure hospitalisations in the high CRP group compared with the low CRP group.

A high CRP level $(>10 \mathrm{mg} / \mathrm{L})$ at discharge from the ADHF hospitalisation was a simple predictor of the 1-year mortality, which might have been partly due to the underlying inflammation that could not be controlled by classical ADHF therapies. Unfortunately, previous studies have failed to demonstrate the efficacy of heart failure-specific treatments targeting inflammation, such as a targeted anticytokine therapy using a TNF- $\alpha$ antagonist, ${ }^{21}$ immune modulation therapy ${ }^{22}$ and statin therapy, ${ }^{23}$ in chronic heart failure. However, a recent randomised controlled trial suggested that an IL-1 $\beta$ inhibitor dose-dependently reduced heart failure hospitalisations and the composite of heart failure hospitalisations or heart failure-related mortality in patients with a previous myocardial infarction and elevated high-sensitivity CRP (hsCRP) levels. ${ }^{24}$ In the setting of ADHF, patients are haemodynamically unstable with marked congestion, which leads to bowel oedema and overgrowth of bacteria flora, resulting in inflammation. ${ }^{25}$ Precipitating inflammation or an infection triggers a haemodynamic collapse due to an increased oxygen demand and surge in the blood pressure in a substantial proportion of patients with ADHF. ${ }^{26}$ Thus, the elevated CRP levels at discharge may reflect a prolonged inflammatory response despite the treatment for ADHF. The efficacy of the therapies to block the upstream inflammation in ADHF has not yet been evaluated, and further study on this topic is warranted.

There is a paucity of data on the association of the CRP level at discharge from an ADHF hospitalisation with the clinical outcomes. A previous study reported that a high CRP level $(\geq 9.5 \mathrm{mg} / \mathrm{L})$ at discharge was associated with an excess adjusted risk for mortality within 120 days; however, this excess risk in the high CRP level patients $(\geq 9.5 \mathrm{mg} / \mathrm{L})$ decreased markedly with time. ${ }^{14}$ Instead, a modestly elevated CRP level $(1.2$ to $9.5 \mathrm{mg} / \mathrm{L}$ ) was associated with an excess adjusted risk for mortality in the long-term follow-up beyond 120 days (median, 510 (IQR, 381 to 765 ) days). ${ }^{14}$ Another previous study reported that the cumulative 3-month incidence of deaths or heart failure hospitalisations was significantly high for a high CRP level (>12.3 mg/L, upper third) at discharge in ADHF patients without a concomitant infection but not in those with a concomitant infection, ${ }^{27}$ however, this was not explicitly adjusted for the confounders. In the present study, a high CRP level $(>10 \mathrm{mg} / \mathrm{L})$ at discharge was clearly associated with an excess adjusted mortality risk at 1 year in $\mathrm{ADHF}$ patients in whom a concomitant infection was excluded as much as possible. Several previous studies have suggested that circulating elevated levels of cytokines and cytokine receptors predict a worse clinical outcome in patients with heart failure. ${ }^{28}{ }^{29}$ Further, a previous study demonstrated that cytokines (TNF- $\alpha$ and IL-6) and their receptors were independent predictors of mortality in patients with advanced heart failure. ${ }^{30}$ IL- 6 works between the upstream of CRP and downstream of interleukin-1 $\beta$ (IL-1 $\beta$ ), and the CRP values are easier to measure than both IL- 6 and IL-1 $\beta$. Therefore, the high CRP level could be a more relevant prognostic predictor in patients with heart failure.

The excess mortality risk in patients with a high CRP at discharge was consistent in all the clinically relevant subgroups. There is a paucity and inconsistency of data of the difference in the CRP levels between ADHF patients with a reduced LVEF and those with a preserved LVEF. ${ }^{11}$ A previous study reported that a decrease of at least $40 \%$ in the hsCRP during hospitalisations (hsCRP levels on admission minus those at discharge) was associated with a low adjusted risk for mortality at 3years in ADHF patients with a preserved LVEF, but not in those with a reduced LVEF. ${ }^{32}$ In the present study, the CRP levels at discharge were not different regardless of a reduced or preserved LVEF. Furthermore, the excess mortality risk in patients with a high CRP at discharge was consistent regardless of a reduced or preserved LVEF, suggesting that the CRP levels on admission might have a different meaning between ADHF patients with a reduced LVEF and those with a preserved LVEF considering the previous report. ${ }^{32}$ Regarding the CRP levels on admission, the excess mortality risk in patients with a high CRP at discharge was consistent regardless of an elevated CRP level on admission. Consistent with the previous studies, ${ }^{33}{ }^{34}$ the CRP levels at discharge were higher in the elderly patients and patients with coronary artery disease. However, the excess mortality risk in patients with a high CRP at discharge was consistent regardless of the age and presence of coronary artery disease. Thus, the high CRP level $(>10 \mathrm{mg} / \mathrm{L})$ at discharge was a prognostic predictor across the wide spectrum of patients with ADHF.

There was no significant excess risk for heart failure hospitalisations in the high CRP group compared with the low CRP group. The association between the CRP levels and severity of systolic dysfunction or symptoms was controversial. ${ }^{65}$ In the present study, there was no significant difference in the proportion of a low LVEF or New York HeartAssociation (NYHA) class III/IV between the high and low CRP groups (table 1). These findings suggest that the association of the elevated CRP levels at discharge from ADHF hospitalisations with the mortality risk at 1 year might not reflect the severity of the systolic dysfunction or symptoms.

\section{Study limitations}

The present study had several limitations. First, we used the CRP values measured in each hospital using a normalsensitivity or high-sensitivity assay, because the present study was based on an observational cohort study in daily clinical practice. This may have caused variability in the measurement of the CRP levels and might have led to an inconsistent classification of the high and low CRP groups. Second, we could not fully address the effects of chronic inflammatory diseases such as autoimmune disease and malignancy on the clinical outcomes. Thus, we conducted subgroup analyses stratified by malignancy, in which we did not find any significant interaction between malignancy and the excess mortality risk in patients with a high CRP. Third, we excluded patients with 
infections using the body temperature and physician's judgement as much as possible. However, there was the possibility of a concomitant infection due to the complex nature of the infection. For example, we could not completely exclude patients who presented without a fever but still had a suspicion of an infection. Fourth, several subgroup analyses had the risk of a multiple comparison as well as a small sample size with a low statistical power. Finally, it is not clear how we could apply the present study findings in clinical practice.

\section{CONCLUSIONS}

A high CRP level $(>10 \mathrm{mg} / \mathrm{L})$ at discharge from an ADHF hospitalisation was associated with an excess mortality risk at 1 year.

\section{Author affiliations}

${ }^{1}$ Department of Cardiology, Hyogo Prefectural Amagasaki General Medical Center, Amagasaki, Japan

${ }^{2}$ Department of Cardiovascular Medicine, Kyoto University Graduate School of Medicine, Kyoto, Japan

${ }^{3}$ Department of Clinical Epidemiology, Hyogo College of Medicine, Nishinomiya, Japan

${ }^{4}$ Department of Cardiology, Shiga Medical Center for Adults, Moriyama, Japan

${ }^{5}$ Division of Cardiology, Tenri Hospital, Tenri, Japan

${ }^{6}$ Department of Cardiovascular Medicine, Kobe City Medical Center General Hospital, Kobe, Japan

${ }^{7}$ Department of Cardiology, National Hospital Organisation Kyoto Medical Center, Kyoto, Kyoto, Japan

${ }^{8}$ Department of Cardiology, Mitsubishi Kyoto Hospital, Kyoto, Kyoto, Japan

${ }^{9}$ Department of Cardiology, Shimabara Hospital, Kyoto, Japan

${ }^{10}$ Department of Cardiology, Japanese Red Cross Otsu Hospital, Otsu, Japan

${ }^{11}$ Department of Cardiology, Hikone Municipal Hospital, Hikone, Japan

${ }^{12}$ Department of Cardiology, Osaka Red Cross Hospital, Osaka, Japan

${ }^{13}$ Department of Cardiology, Kishiwada City Hospital, Kishiwada, Japan

${ }^{14}$ Department of Cardiology, Kansai Electric Power Hospital, Osaka, Japan

${ }^{15}$ Department of Cardiology, Shizuoka General Hospital, Shizuoka, Japan

${ }^{16}$ Department of Cardiology, Kurashiki Central Hospital, Kurashiki, Japan

${ }^{17}$ Department of Cardiology, Kokura Memorial Hospital, Kitakyushu, Japan

${ }^{18}$ Department of Cardiology, Japanese Red Cross Wakayama Medical Center, Wakayama, Japan

${ }^{19}$ Department of Cardiology, Nishi-Kobe Medical Center, Kobe, Japan

${ }^{20}$ Cardiovascular Center, The Tazuke Kofukai Medical Research Institute, Kitano

Hospital, Osaka, Japan

${ }^{21}$ Department of Cardiovascular Medicine, Shinshu University Graduate School of Medicine, Matsumoto, Japan

Acknowledgements We appreciate the support and collaboration of the co-investigators participating in the KCHF Registry. We also would like to express our gratitude to Mr John Martin for his grammatical assistance.

Contributors YNi and TKat had full access to all of the data in the study and take responsibility for the integrity of the data and accuracy of the data analysis. Concept and design: YNi, TKat, TMorim, HY, YI, YT, EY, YY, TKit, RT, Mlg, MKat, MTa, TJ, TI, KN, TKaw, AK, RN, YK, TMorin, KS, MKaw, YSe, Mln, MTo, YSa and TKim. Acquisition, analysis or interpretation of data: YNi, TKat, HY, YI, YT, EY, YY, TKit, RT, Mlg, MKat, MTa, TJ, TI, KN, TKaw, AK, RN, YK, TMorin, KS, MKaw, YSe, Mln, MTo, YF, YNa, KA, $\mathrm{KKa}, \mathrm{SS}, \mathrm{KO}, \mathrm{KKu}, \mathrm{NO}$ and YSa. Drafting of the manuscript: YNi, TKat, HY and TKim. Critical revision of the manuscript for important intellectual content: YNi, TKat, HY, TKit and TKim. Statistical analysis: YNi, TKat and TMorim. Administrative, technical or material support: TKat, YSa and TKim. Supervision: TKat, TMorim, HY, YF, YNa, KA, KKa, SS, KO, KKu, NO, YSa and TKim.

Funding This study was supported by a grant from the Japan Agency for Medical Research and Development (18059186) to TKat, KKu and NO. The founder had no role in the study design, collection, analysis or interpretation of the data, writing the manuscript or the decision to submit the paper for publication.

Competing interests None declared.
Patient consent for publication Not required.

Ethics approval The study was approved by the institutional review boards of Kyoto University Graduate School of Medicine (approval number: E2311), Shiga General Hospital (approval number: 20141120-01), Tenri Hospital (approval number: 640), Kobe City Medical Center General Hospital (approval number: 14094), Hyogo Prefectural Amagasaki General Medical Center (approval number: Rinri 26-32), National Hospital Organisation Kyoto Medical Center (approval number: 14-080), Mitsubishi Kyoto Hospital (approved 11/12/2014), Okamoto Memorial Hospital (approval number: 201503), Japanese Red Cross Otsu Hospital (approval number: 318), Hikone Municipal Hospital (approval number: 26-17), Japanese Red Cross Osaka Hospital (approval number: 392), Shimabara Hospital (approval number: E2311), Kishiwada City Hospital (approval number: 12), Kansai Electric Power Hospital (approval number: 26-59), Shizuoka General Hospital (approval number: Rin14-11-47), Kurashiki Central Hospital (approval number: 1719), Kokura Memorial Hospital (approval number: 14111202), Kitano Hospital (approval number: P14-11012) and Japanese Red Cross Wakayama Medical Center (approval number: 328). A waiver of written informed consent from each patient was granted by the institutional review boards of Kyoto University and each participating centre.

Provenance and peer review Not commissioned; externally peer reviewed.

Data availability statement All data relevant to the study are included in the article or uploaded as supplementary information. All data relevant to the study are included in the article or uploaded as supplementary information. No additional data available.

Supplemental material This content has been supplied by the author(s). It has not been vetted by BMJ Publishing Group Limited (BMJ) and may not have been peer-reviewed. Any opinions or recommendations discussed are solely those of the author(s) and are not endorsed by BMJ. BMJ disclaims all liability and responsibility arising from any reliance placed on the content. Where the content includes any translated material, BMJ does not warrant the accuracy and reliability of the translations (including but not limited to local regulations, clinical guidelines, terminology, drug names and drug dosages), and is not responsible for any error and/or omissions arising from translation and adaptation or otherwise.

Open access This is an open access article distributed in accordance with the Creative Commons Attribution Non Commercial (CC BY-NC 4.0) license, which permits others to distribute, remix, adapt, build upon this work non-commercially, and license their derivative works on different terms, provided the original work is properly cited, appropriate credit is given, any changes made indicated, and the use is non-commercial. See: http://creativecommons.org/licenses/by-nc/4.0/.

\section{ORCID iDs}

Takao Kato http://orcid.org/0000-0001-8213-7999

Takeshi Morimoto http://orcid.org/0000-0002-6844-739X

Moritake Iguchi http://orcid.org/0000-0001-5029-1203

Kazuya Nagao http://orcid.org/0000-0002-5043-8778

Satoshi Shizuta http://orcid.org/0000-0003-4587-9031

Takeshi Kimura http://orcid.org/0000-0002-5665-4076

\section{REFERENCES}

1 Kurmani S, Squire I. Acute heart failure: definition, classification and epidemiology. Curr Heart Fail Rep 2017;14:385-92.

2 Maggioni AP, Dahlström U, Filippatos G, et al. EURObservational research programme: regional differences and 1-year follow-up results of the heart failure pilot survey (ESC-HF Pilot). Eur J Heart Fail 2013;15:808-17.

3 Tavazzi L, Senni M, Metra M, et al. Multicenter prospective observational study on acute and chronic heart failure: one-year follow-up results of IN-HF (Italian network on heart failure) outcome registry. Circ Heart Fail 2013;6:473-81.

4 Mendall MA, Patel P, Asante M, et al. Relation of serum cytokine concentrations to cardiovascular risk factors and coronary heart disease. Heart 1997;78:273-7.

5 Anker SD, von Haehling S. Inflammatory mediators in chronic heart failure: an overview. Heart 2004;90:464-70.

6 Anand IS, Latini R, Florea VG, et al. C-reactive protein in heart failure: prognostic value and the effect of valsartan. Circulation 2005;112:1428-34.

7 Windram JD, Loh PH, Rigby AS, et al. Relationship of high-sensitivity C-reactive protein to prognosis and other prognostic markers in outpatients with heart failure. Am Heart J 2007;153:1048-55.

8 Pellicori P, Zhang J, Cuthbert J, et al. High-sensitivity C-reactive protein in chronic heart failure: patient characteristics, phenotypes, and mode of death. Cardiovasc Res 2020;116:91-100. 
9 Alonso-Martínez JL, Llorente-Diez B, Echegaray-Agara M, et al. $\mathrm{C}$-reactive protein as a predictor of improvement and readmission in heart failure. Eur J Heart Fail 2002;4:331-6.

10 Siirilä-Waris K, Lassus J, Melin J, et al. Characteristics, outcomes, and predictors of 1-year mortality in patients hospitalized for acute heart failure. Eur Heart $J$ 2006;27:3011-7.

11 Minami Y, Kajimoto K, Sato N, et al. C-reactive protein level on admission and time to and cause of death in patients hospitalized for acute heart failure. Eur Heart J Qual Care Clin Outcomes 2017;3:148-56.

12 Yamamoto E, Kato T, Ozasa N, et al. Kyoto congestive heart failure (KCHF) study: rationale and design. ESC Heart Fail 2017;4:216-23.

13 Yaku H, Ozasa N, Morimoto T, et al. Demographics, management, and in-hospital outcome of hospitalized acute heart failure syndrome patients in contemporary real clinical practice in japan- observations from the prospective, multicenter kyoto congestive heart failure (KCHF) registry. Circ J 2018;82:2811-9.

14 Minami Y, Kajimoto K, Sato N, et al. Effect of elevated C-reactive protein level at discharge on long-term outcome in patients hospitalized for acute heart failure. Am J Cardiol 2018;121:961-8

15 Ichihara K, Yomamoto Y, Hotta T, et al. Collaborative derivation of reference intervals for major clinical laboratory tests in Japan. Ann Clin Biochem 2016:53:347-56.

16 Kappetein AP, Head SJ, Généreux P, et al. Updated standardized endpoint definitions for transcatheter aortic valve implantation: the valve academic research Consortium-2 consensus document. J Am Coll Cardiol 2012;60:1438-54

17 Kitai T, Miyakoshi C, Morimoto T, et al. Mode of death among Japanese adults with heart failure with preserved, Midrange, and reduced ejection fraction. JAMA Netw Open 2020;3:e204296.

18 Su K, Kato T, Toyofuku M, et al. Association of previous hospitalization for heart failure with increased mortality in patients hospitalized for acute decompensated heart failure. Circulation Reports 2019:1:517-24.

19 Yaku H, Kato T, Morimoto T, et al. Risk factors and clinical outcomes of functional decline during hospitalisation in very old patients with acute decompensated heart failure: an observational study. BMJ Open 2020;10:e032674.

20 Yaku H, Kato T, Morimoto T, et al. Association of mineralocorticoid receptor antagonist use with all-cause mortality and hospital readmission in older adults with acute decompensated heart failure. JAMA Netw Open 2019;2:e195892.

21 Mann DL, McMurray JJV, Packer M, et al. Targeted anticytokine therapy in patients with chronic heart failure: results of the randomized etanercept worldwide evaluation (renewal). Circulation 2004;109:1594-602.

22 Torre-Amione G, Anker SD, Bourge RC, et al. Results of a nonspecific immunomodulation therapy in chronic heart failure
(ACCLAIM trial): a placebo-controlled randomised trial. Lancet 2008:371:228-36.

23 Tavazzi L, Maggioni AP, Marchioli R, et al. Effect of rosuvastatin in patients with chronic heart failure (the GISSI-HF trial): a randomised, double-blind, placebo-controlled trial. Lancet 2008;372:1231-9.

24 Everett BM, Cornel JH, Lainscak M, et al. Anti-inflammatory therapy with canakinumab for the prevention of hospitalization for heart failure. Circulation 2019;139:1289-99.

25 Valentova M, von Haehling S, Bauditz J, et al. Intestinal congestion and right ventricular dysfunction: a link with appetite loss, inflammation, and cachexia in chronic heart failure. Eur Heart $J$ 2016;37:1684-91.

26 Arrigo M, Gayat E, Parenica J, et al. Precipitating factors and 90-day outcome of acute heart failure: a report from the intercontinental great registry. Eur J Heart Fail 2017;19:201-8.

27 Lourenço P, Paulo Araújo J, Paulo C, et al. Higher C-reactive protein predicts worse prognosis in acute heart failure only in noninfected patients. Clin Cardiol 2010;33:708-14.

28 Ferrari R, Bachetti T, Confortini R, et al. Tumor necrosis factor soluble receptors in patients with various degrees of congestive heart failure. Circulation 1995;92:1479-86.

29 Tsutamoto T, Hisanaga T, Wada A, et al. Interleukin-6 spillover in the peripheral circulation increases with the severity of heart failure, and the high plasma level of interleukin- 6 is an important prognostic predictor in patients with congestive heart failure. J Am Coll Cardiol 1998;31:391-8

30 Deswal A, Petersen NJ, Feldman AM, et al. Cytokines and cytokine receptors in advanced heart failure: an analysis of the cytokine database from the Vesnarinone trial (vest). Circulation 2001;103:2055-9.

31 Tromp J, Khan MAF, Klip IJsbrandT, et al. Biomarker profiles in heart failure patients with preserved and reduced ejection fraction. $J \mathrm{Am}$ Heart Assoc 2017;6 doi:10.1161/JAHA.116.003989

32 Lourenço P, Pereira J, Ribeiro A, et al. C-reactive protein decrease associates with mortality reduction only in heart failure with preserved ejection fraction. J Cardiovasc Med 2019;20:23-9.

33 Rosa GM, Scagliola R, Ghione P, et al. Predictors of cardiovascular outcome and rehospitalization in elderly patients with heart failure. Eur J Clin Invest 2019;49:e13044.

34 Kiuchi S, Hisatake S, Kabuki T, et al. Cardio-ankle vascular index and $\mathrm{C}$-reactive protein are useful parameters for identification of ischemic heart disease in acute heart failure patients. J Clin Med Res 2017;9:439-45.

35 van Boven N, Akkerhuis KM, Anroedh SS, et al. In search of an efficient strategy to monitor disease status of chronic heart failure outpatients: added value of blood biomarkers to clinical assessment. Neth Heart J 2017;25:634-42. 